\title{
Kombiniert-heterozygote Defizienz von Komplementfaktor C7 bei einer Patientin mit rezidivierender Meningitis
}

\author{
Rebecca Schirinzi ${ }^{1}$, Jean Pierre Lantin ${ }^{2}$, Véronique Frémeaux-Bacchi ${ }^{3}$, Jürg A. Schifferli ${ }^{1}$, Marten Trendelenburg ${ }^{1,4}$
}

\section{ZUSAMMENFASSUNG}

$\square$ Hintergrund: Die Assoziation zwischen Komplementdefizienzen, insbesondere von Komponenten der terminalen Kaskade (C5-C9), und dem Auftreten von Meningokokkeninfekten und bakteriellen Meningitiden ist gut beschrieben.

$\square$ Fallbeschreibung: In dem vorliegenden Fallbericht wird dabei erstmals ein kombiniert-heterozygoter Defekt im C7-Gen beschrieben, der noch eine Restproduktion von C7 erlaubte. Diese Restproduktion reichte jedoch nicht aus, um vor rezidivierenden Meningitiden zu schützen.

Schlussfolgerung: Der Fallbericht zeigt erneut den Stellenwert der Komplementdiagnostik bei Patienten mit Meningokokkeninfekt und die Notwendigkeit, auch Patienten mit reduzierter, aber noch messbarer Komplementaktivität einer weiteren Abklärung auf eine Komplementdefizienz zuzuführen.

Schlüsselwörter: Komplement $\cdot$ Defizienz $\cdot$ Meningitis

Med Klin 2006;101:655-8. DOI 10.1007/s00063-006-1070-4

\section{ABSTRACT}

Combined-Heterozygous Deficiency of Complement C7 in a Patient with Recurrent Meningitis

Background: The association between complement deficiencies and the increased risk for meningococcal infections and bacterial meningitis is well described and most striking in patients with deficiencies of one of the late complement components, i.e., C5-C9.

$\square$ Case Report: In the presented study the first case of a patient with combined-heterozygous deficiency of complement C7 is described. The defect led to a strongly reduced but still measurable production of $\mathrm{C} 7$. However, the low concentration of $\mathrm{C} 7$ was not protective against recurrent bacterial meningitis.

$\square$ Conclusion: The reported case illustrates once again the necessity of complement analysis in patients with meningitis. Not only patients with undetectable complement activity but also those with strongly reduced but still measurable complement function should be analyzed for a possible complement deficiency.

Key Words: Complement $\cdot$ Meningitis · Deficiency

Med Klin 2006;101:655-8. DOI 10.1007/s00063-006-1070-4

${ }^{1}$ Innere Medizin B, Universitätsspital Basel, Schweiz,

${ }^{2}$ Pregassona, Schweiz,

${ }^{3}$ Service d'Immunologie Biologique, Hôpital Européen Georges Pompidou, Paris, Frankreich,

${ }^{4}$ Labor für Klinische Immunologie, Departement Forschung, Universitätsspital Basel, Schweiz.

Eingang des Manuskripts: 30. 1. 2006.

Annahme des überarbeiteten Manuskripts: 7. 4. 2006.
R ei genetisch bedingten Defizienzen von Komplementfaktoren handelt es sich meist um autosomal-rezessive Erbkrankheiten. Heterozygote Patienten produzieren dabei eine annähernd normale Menge des betroffenen Komplementproteins. Häufungen bestimmter Krankheiten werden vor allem bei Patienten beobachtet, deren betroffenes Komplementprotein stark erniedrigt oder nicht messbar ist, wie dies bei homozygoten oder kombiniert-heterozygoten Defekten beobachtet wird.

Defizienzen der terminalen Komplementkomponenten führen vermehrt zu Infekten durch Neisserien, insbesondere Neisseria meningitidis. In der vorliegenden Fallbeschreibung berichten wir über eine junge Patientin mit rezidivierenden bakteriellen Meningitiden. Ursächlich fand sich dabei eine hier erstmals beschriebene kombiniert-heterozygote Defizienz von Komplementfaktor C7.

\section{Fallbeschreibung}

$\square$ Anamnese: Die 22-jährige Patientin wurde im August 2002 wegen eines Status febrilis mit Cephalea, Übelkeit und leichter psychomotorischer Verlangsamung hospitalisiert. Sie berichtete über einen seit 5 Tagen bestehenden grippalen Infekt mit Fieber bis $39^{\circ} \mathrm{C}$. Chronische Erkrankungen und eine regelmäßige Medikamenteneinnahme wurden verneint. Die Patientin berichtete jedoch von einer im Alter von 19 Jahren aufgetretenen bakteriellen Meningitis mit Waterhouse-Friderichsen-Syndrom. Es handelte sich dabei um einen Infekt mit Neisseria meningitidis der Serogruppe W135, von dem sie sich vollständig erholte. Die übrige Vorgeschichte war bland. In der Familienanamnese fiel auf, dass auch der Vater der Patientin dreimalig eine Meningitis gehabt hatte. Die Erreger dieser Meningitisepisoden waren retrospektiv nicht mehr zu ermitteln. 
Klinischer Aufnahmebefund: Bei der Aufnahme war die Patientin leicht somnolent und febril, jedoch in einem guten Allgemeinzustand. Der Glasgow-Coma-Score lag bei 15. Der Blutdruck betrug $80 / 60 \mathrm{mmHg}$, die Pulsfrequenz $100 / \mathrm{min}$ und die periphere Sauerstoffsättigung 93\%. Bis auf einen leichten Meningismus war die Patientin im übrigen internistischen und neurologischen Status unauffällig. Insbesondere bestanden keine Petechien und keine Photo- oder Phonophobie.

$\square$ Laborbefunde: Im Blutbild fanden sich eine normozytär-normochrome Anämie mit einem Hämoglobin von 10,3 g/dl (Norm 12,0-16,0 g/dl) und eine Leukozytose von $40500 / \mu$ l (Norm 4 000-10 000/ $\mu \mathrm{l})$ mit relativer Lymphopenie (6\%) und Linksverschiebung (55\% stabkernige Granulozyten). AuBerdem zeigten sich eine Thrombozytopenie von $131000 / \mu$ l (Norm

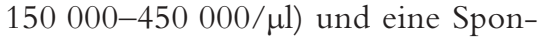
tan-INR (International Normalized Ratio) von 1,6. Das C-reaktive Protein betrug 131,1 mg/dl (Norm $<5$ $\mathrm{mg} / \mathrm{dl})$. Die weiteren laborchemischen Parameter wie Elektrolyte, Lactat, Nieren- und Leberwerte waren bis auf eine leicht erhöhte Alaninaminotransferase $(55 \mathrm{U} / 1$, Norm < $37 \mathrm{U} / 1)$ unauffällig.

$\square$ Ergänzende Untersuchungen: Die Liquoranalyse war vereinbar mit einer bakteriellen Meningitis (makroskopisch trüber Liquor mit 1280 Leukozyten/ $\mu l$, vorwiegend neutrophile Granulozyten), jedoch konnten weder im Direktpräparat noch in der Kultur Erreger nachgewiesen werden. Auch die Blutkulturen blieben steril.

Nach Abheilung der Meningitisepisode und Entlassung stellte sich die Patientin zur immunologischen Abklärung vor. Ein Kontrollblutbild war jetzt unauffällig, ein Immunglobulinmangel (IgG, IgM, IgA) oder eine IgG-Subklassendefizienz ließ sich nicht feststellen. Außerdem fanden sich nach Impfung protektive IgG-Antikörper-Titer gegen Tetanus, Diphtherie, Haemophilus influenzae und Pneumokokken. Allerdings fiel ein deutlich erniedrigter Wert beim Komplementfunktionstest (CH50) von 23\% (Norm > 70\%) auf. Im Wissen, dass rezidivierende $\mathrm{Me}$ ningitiden gehäuft bei Defizienzen

Tabelle 1. Komplementwerte der Patientin und ihrer Familie.

\begin{tabular}{|lrrrrr|}
\hline & Patientin & Vater & Mutter & \multicolumn{2}{c|}{ Schwester Normwerte } \\
& & & & & \\
CH50 (\%) & 23 & 0 & 88 & 90 & $70-130$ \\
C3-Antigen (mg/l) & 881 & 1390 & 803 & 1060 & $660-1250$ \\
C4-Antigen (mg/l) & 181 & 235 & 170 & 158 & $93-380$ \\
C5-Antigen (\%) & 112 & 123 & 114 & 92 & $60-140$ \\
C6-Antigen (\%) & 120 & 103 & 122 & 74 & $60-140$ \\
C7-Antigen (\%) & 1 & $<0,1$ & 65 & 60 & $60-140$ \\
C8-Antigen (\%) & 132 & 135 & 145 & 89 & $60-140$ \\
C9-Antigen (\%) & 131 & 98 & 107 & 88 & $60-140$ \\
\hline
\end{tabular}

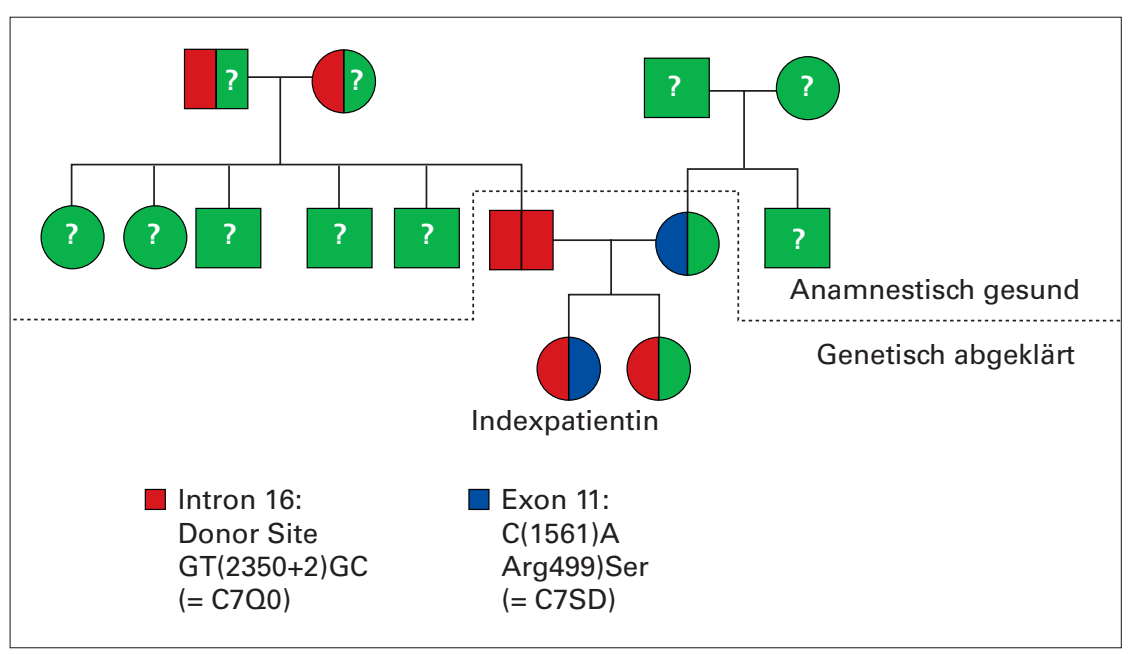

Abbildung 1. Stammbaum der Patientenfamilie bezüglich C7-Defizienzen. Die Verwandschaft zweiten Grades der Patientin wurde genetisch nicht untersucht, Meningitisepisoden wurden jedoch nicht beschrieben. Diese klinische Beobachtung schließt weitere heterozygote oder auch homozygote Komplementdefizienzen bei den Angehörigen nicht aus.

der terminalen Komplementfaktoren C5-C9 vorkommen, wurde daher eine eingehende Analyse des Komplementsystems der Patientin, ihrer fast gleich alten Schwester und ihrer Eltern veranlasst.

Bei der Patientin zeigte sich dabei in der antigenetischen Bestimmung der Komplementfaktoren ein deutlich erniedrigter Wert für C7 (1\% der Norm). Diese geringe Restmenge an C7 hatte ausgereicht, um 23\% der hämolytischen Komplementfunktion (CH50) aufrechtzuerhalten. Eine ähnliche, aber noch ausgeprägtere Konstellation mit nicht mehr nachweisbarem $\mathrm{CH} 50$ und C7 fand sich beim Vater der Patientin ( $0 \%$ bzw. < 0,1\% der Norm), während die Schwester und die Mutter nur leicht verminderte Konzentrationen an C7 mit noch normalem CH50 aufwiesen. Die Ergebnisse sind in Tabelle $1 \mathrm{zu}-$ sammengefasst. Aufgrund dieser Wer- te erfolgte eine molekularbiologische Untersuchung, welche die C7-Defizienzen bestätigte. Interessanterweise fand sich dabei beim Vater der Patientin ein homozygoter Defekt auf dem Gen für C7 in Intron 16 [Donor Site GT(2350+2)GC, entsprechend C7Q0], während bei der Mutter ein anderer, heterozygoter Defekt in Exon 11 vorlag [C(1561)A, entsprechend C7SD]. Bei der Patientin war es daraus zu einem kombiniert-heterozygoten Defekt (C7Q0/C7SD) gekommen, während die Schwester nur heterozygot für die vom Vater geerbte Mutation war (Abbildung 1).

\section{Therapie und Verlauf}

Unter empirischer antibiotischer Therapie heilte die Meningitis komplikationslos aus. Bei der Patientin und ihrem Vater wurde eine prophylaktische 
Meningokokken- und Pneumokokkenimpfung durchgeführt. Außerdem wurde empfohlen, bei Auftreten von Fieber sofort eine antibiotische Therapie mit Amoxicillin zu beginnen. Weitere Meningitisepisoden wurden bislang ( $>3$ Jahre nach der geschilderten Episode) nicht beobachtet.

\section{DISKUSSION}

Bei der hier vorgestellten Patientin liegt eine erstmals beschriebene kombiniert-heterozygote Defizienz von C7 vor. Aus den Untersuchungsergebnissen lässt sich ableiten, dass die von der Mutter vererbte Mutation C7SD noch eine geringe Restproduktion von C7 erlaubt, während es bei der vom Vater vererbten Mutation C7Q0 zu einem vollständigen Erliegen der Produktion kommt. Die geringe Restproduktion von $\mathrm{C} 7$ bei der Patientin erlaubt eine noch messbare hämolytische Funktion der Komplementkaskade (CH50), reicht aber offensichtlich nicht aus, um vor Infekten mit Meningokkoken zu schützen.

Das Komplementsystem stellt einen wichtigen Abwehrmechanismus gegen Infekte durch Neisserien dar, die trotz guter diagnostischer und therapeutischer Möglichkeiten aufgrund des Risikos für einen septischen Schock eine Mortalität von bis zu 40\% haben [1]. Patienten mit bekannter Defizienz der terminalen Komplementfaktoren neigen zu rezidivierenden Infekten durch Meningokokken. Die Gesamthäufigkeit hereditärer Komplementdefizienzen bei Patienten mit Status nach nicht näher differenzierter bakterieller $\mathrm{Me}-$ ningitis ist sehr variabel und beträgt je nach Studie zwischen 1\% und 25\% [2-5]. Untersuchungen an Patienten mit Zustand nach bewiesener Meningokokkenmeningitis zeigten z.T. sogar eine Prävalenz der Komplementdefizienzen von bis zu 50\%, insbesondere bei Patienten mit rezidivierender Erkrankung wie in dem hier beschriebenen Fall [4, 6-11]. Diskrepanzen zwischen den teilweise sehr unterschiedlichen Studienergebnissen lassen sich dabei u.a. auf verschiedene Rekrutierungsmethoden, unterschiedliche Diagnostik und genetische Unterschiede der Patientenkollektive zurückführen.

Auch bei gesunden Familienangehörigen betroffener Patienten sind
Komplementdefizienzen gut beschrieben. Daraus lässt sich ableiten, dass eine Komplementdefizienz nicht zwingend zu einer Meningitis führen muss [12-14]. Außerdem fällt auf, dass komplementdefiziente Patienten überdurchschnittlich häufig Infekte durch eher seltene Serogruppen wie X, Y oder W135 hatten, das Alter bei Erstmanifestation höher als bei komplementsuffizienten Patienten war und in bis $\mathrm{zu} 40 \%$ Rezidive auftraten [1, 6 , 15].

Aufgrund der o.g. Punkte ist es empfehlenswert, bei allen jungen $\mathrm{Pa}$ tienten mit Meningitis eine Komplementabklärung mittels Bestimmung des CH50 durchzuführen, insbesondere bei rezidivierenden Episoden oder positiver Familienanamnese. Wegen des häufig ausgeprägten Komplementverbrauchs im Akutstadium der Erkrankung sollte diese Untersuchung erst nach Ausheilen der Episode erfolgen. Neben dem gut etablierten CH50-Test ist jetzt auch eine ELISA-basierte Methode verfügbar, mit der alle drei Aktivierungswege der Komplementkaskade zuverlässig funktionell untersucht werden können [16]. Bei Defizienzen der terminalen Komplementkomponenten kommt es dabei zu einer verminderten oder sogar ganz aufgehobenen Aktivität aller drei Aktivierungswege. Ein solcher Befund sollte eine weitergehende Abklärung auf eine Komplementdefizienz nach sich ziehen und auch Verwandte ersten Grades einschließen [1, 17-19]. Neben der rein quantitativen, also antigenetischen Bestimmung von Komplementfaktoren ist es auch möglich, diese funktionell, z.B. durch Resubstitution mit gereinigten Einzelkomponenten, zu bestimmen.

Ist die Diagnose gestellt, erachten wir eine gründliche Aufklärung der Patienten für wichtig, damit diese bei Infektzeichen richtig reagieren und ggf. einen Arzt oder ein Krankenhaus aufsuchen [1, 18]. Mittel der Wahl bei komplementdefizienten Patienten mit gesichertem Meningokokkeninfekt ist wie bei komplementsuffizienten Patienten Penicillin G, bei Resistenzen und/oder Penicillinallergie meist ein Cephalosporin der dritten Generation. Nach Abheilung erscheint unseres Erachtens wegen der Häufung atypischer Serogruppen eine prophylaktische
Impfung gegen Meningokokken und auch gegen Pneumokokken gerechtfertigt [1, 7, 20]. Zusätzlich erhielten unsere Patientin und ihr Vater eine Stand-by-Medikation mit Amoxicillin für den Fall eines fieberhaften Infekts.

\section{SCHLUSSFOLGERUNG}

Bei allen jungen Patienten mit $\mathrm{Me}-$ ningokokkenmeningitis sollte eine Komplementdefizienz in Betracht gezogen und aktiv gesucht werden. Bereits eine deutlich verminderte Funktion der Komplementkaskade (CH50) sollte eine eingehende Abklärung unter Einschluss der nächsten Verwandten nach sich ziehen. Durch eine gute $\mathrm{Pa}-$ tientenaufklärung und prophylaktische Impfungen können so Rezidive vermieden bzw. frühzeitig therapiert werden.

\section{Literatur}

1. Debard AL, Lamy B, Monneret G, et al. FcgammaRIIIb and complement component C7 codeficiency in a patient with recurrence of fulminant meningococcal septic shock. Clin Infect Dis 2005;40: 1679-83.

2. Fijen CA, Kuijper EJ, Tjia HG, et al. Complement deficiency predisposes for meningitis due to nongroupable meningococci and Neisseria-related bacteria. Clin Infect Dis 1994;18:780-4.

3. Maitra S, Ghosh SK. Recurrent pyogenic meningitis - a retrospective study. Q J Med 1989;73: 919-29.

4. Platonov AE, Beloborodov VB, Vershinina IV Meningococcal disease in patients with late complement component deficiency: studies in the U.S.S.R. Medicine (Baltimore) 1993;72:374-92.

5. Ross SC, Densen P. Complement deficiency states and infection: epidemiology, pathogenesis and consequences of neisserial and other infections in an immune deficiency. Medicine (Baltimore) 1984;63: 243-73.

6. Fijen CA, Kuijper EJ, Hannema AJ, et al. Complement deficiencies in patients over ten years old with meningococcal disease due to uncommon serogroups. Lancet 1989;2:585-8.

7. D'Amelio R, Agostoni A, Biselli R, et al. Complement deficiency and antibody profile in survivors of meningococcal meningitis due to common serogroups in Italy. Scand J Immunol 1992;35: 589-95.

8. Nishizaki M. The association between deficiency of terminal complement components and the occurrence of meningococcal meningitis. Fukuoka Igaku Zasshi 1992;83:201-8

9. Schlesinger M, Nave Z, Levy Y, et al. Prevalence of hereditary properdin, $\mathrm{C} 7$ and $\mathrm{C} 8$ deficiencies in patients with meningococcal infections. Clin Exp Immunol 1990;81:423-7.

10. Fijen CA, Kuijper EJ, Te Bulte MT, et al. Assessment of complement deficiency in patients with meningococcal disease in the Netherlands. Clin Infect Dis 1999;28:98-105.

11. Ellison RT 3rd, Kohler PF, Curd JG, et al. Prevalence of congenital or acquired complement deficiency in patients with sporadic meningococcal disease. N Engl J Med 1983;308:913-6.

12. Delage JM, Bergeron P, Simard J, et al. Hereditary C7 deficiency. Diagnosis and HLA studies in a 
French-Canadian family. J Clin Invest 1977;60: 1061-9.

13. Lee TJ, Utsinger PD, Snyderman R, et al. Familial deficiency of the seventh component of complement associated with recurrent bacteremic infections due to Neisseria. J Infect Dis 1978;138: 359-68.

14. Wellek B, Opferkuch W. A case of deficiency of the seventh component of complement in man. Biological properties of a C7-deficient serum and description of a C7-inactivating principle. Clin Exp Immunol 1975;19:223-35.

15. Zimran A, Rudensky B, Kramer MR, et al. Hereditary complement deficiency in survivors of meningococcal disease: high prevalence of $\mathrm{C} 7 / \mathrm{C} 8$ deficiency in Sephardic (Moroccan) Jews. Q J Med 1987;63:349-58.

16. Seelen MA, Roos A, Wieslander J, et al. Functional analysis of the classical, alternative, and MBL pathways of the complement system: standardization and validation of a simple ELISA. J Immunol Methods 2005;296:187-98.

17. Angibaud G, Durand G, Rascol A, et al. Recurrent Neisseria meningitidis meningitis associated with homozygote complement C7 fraction deficiency. Rev Neurol (Paris) 1992;148:237-8.

18. Corvini M, Randolph C, Aronin SI. Complement C7 deficiency presenting as recurrent aseptic meningitis. Ann Allergy Asthma Immunol 2004;93: $200-5$
19. Rameix-Welti MA, Chedani H, Blouin J, et al. Neisseria meningitidis infection. Clinical criteria orienting towards a deficiency in the proteins of the complement. Presse Med 2005;34:425-30.

20. Schlesinger M, Greenberg R, Levy J, et al. Killing of meningococci by neutrophils: effect of vaccination on patients with complement deficiency. J Infect Dis 1994;170:449-53.

\section{Korrespondenzanschrift}

Dr. Marten Trendelenburg

Innere Medizin B/

Klinische Immunologie

Universitätsspital Basel

Petersgraben 4

4031 Basel

Schweiz

Telefon (+41/61) 265-2525

Fax -4759

E-Mail:marten.trendelenburg@unibas.ch 